\title{
Pressure-Induced Delocalization of Photoexcited States in a Semiconducting Polymer
}

\author{
Sebastian Albert-Seifried, ${ }^{1, *}$ Justin M. Hodgkiss, ${ }^{1, \dagger}$ Frédéric Laquai, ${ }^{1,}$ Hugo A. Bronstein, ${ }^{2}$ \\ Charlotte K. Williams, ${ }^{2}$ and Richard H. Friend ${ }^{1, \S}$ \\ ${ }^{1}$ Cavendish Laboratory, Department of Physics, University of Cambridge, CB3 OHE, United Kingdom \\ ${ }^{2}$ Department of Chemistry, Imperial College London, London SW7 2AZ, United Kingdom
}

(Received 4 August 2010; published 2 November 2010)

\begin{abstract}
We present broadband transient absorption spectroscopy on the fluorescent copolymer poly $(9,9$ dioctylfluorene-co-benzothiadiazole) under hydrostatic pressure of up to $75 \mathrm{kbar}$. We observe a strong reduction of the stimulated emission intensity under pressure, coupled with slower decay kinetics and reduced fluorescence intensity. These observations indicate increased delocalization of photogenerated singlet excitons, facilitated by an increased dielectric constant at high pressure. Spin triplet excitons, generated via an iridium complex-F8BT oligomer, show reduced lifetimes under pressure.
\end{abstract}

DOI: 10.1103/PhysRevLett.105.195501

Organic semiconductors have much lower dielectric constants $(\varepsilon \sim 2-4)$ than inorganic semiconductors $(\varepsilon \sim 11-12$ for silicon), and this controls many of their properties. Thus, photoexcitations take the form of tightly bound Frenkel excitons, and exciton binding energies $(0.2-1 \mathrm{eV})$ far exceed those in most inorganic semiconductors. Organic semiconductors also exhibit large singlettriplet exchange energies $(\sim 0.4-0.7 \mathrm{eV})$ [1]. In organic photovoltaic (OPV) cells, a large driving force is required to favor efficient dissociation of these tightly bound optical excitations. This is achieved at donor-acceptor heterointerfaces [2-4] at the expense of open-circuit voltage. Here we show that the dielectric constant, and hence many of the electronic properties, can be changed by subjecting a polymer film to high hydrostatic pressure. The polymer selected for this study is poly(9,9-di-octylfluorene-cobenzothiadiazole) (F8BT) (Fig. 1), a highly fluorescent donor-acceptor copolymer [5] that is widely used for polymer light-emitting diodes and OPVs [6,7]. To estimate the effect of pressure on the dielectric constant $(\varepsilon)$ we see how two factors, the electron density $(N)$ and the resonance frequency $\left(\omega_{0}\right)$, affect the dielectric response for the Lorentz oscillator model. We can express the lowfrequency limit for the dielectric constant as

$$
\varepsilon_{r}(0) \equiv \varepsilon_{\mathrm{st}}=1+\chi+\frac{N e^{2}}{\epsilon_{0} m \omega_{0}^{2}},
$$

where $m$ is the reduced mass, $N$ is the electron density in the valence band, and $\omega_{0}$ is their resonance frequency characteristic of the semiconductor band gap. We assess $\omega_{0}$ from the onset of the absorption band and associated photoluminescence. We observe below that $N$ and $\omega_{0}$ will both change with increasing pressure so as to raise $\varepsilon$. Electronic contributions with higher resonance frequencies are represented by $\chi$ but are not further considered here.

Compressibility studies on organic semiconductors have reported similar values for molecular crystals and conjugated polymers. Oligo(paraphenylene)s show a
PACS numbers: 81.05.Fb, 62.50.-p, 71.20.Rv, 78.47.jb

volume strain of $\sim 25 \%$ over $60 \mathrm{kbar}$ [8]. X-ray diffraction measurements on poly(octyl-thiophene) also show a volume strain of 20\%-30\% over $80 \mathrm{kbar}$ [9]. The compressibility along the $\pi-\pi$ stacking direction and along the side chains generally far exceeds that along the polymer backbone. As no experimental data are available for F8BT, we assume a volume strain of $25 \%$ over $75 \mathrm{kbar}$ for our discussion. The photoluminescence (PL) spectra that we will discuss later show a shift of the PL onset from $500 \mathrm{~nm}$ to $\sim 620 \mathrm{~nm}$ at high pressure, and hence $\omega_{0}$ is reduced by $20 \%$ at $75 \mathrm{kbar}$. These two effects increase $\varepsilon$ from 2.7 [10] to about 4.5 at a pressure of $75 \mathrm{kbar}$. This increase will certainly modify the properties of excitons under pressure. The upper limit to the exciton binding energy for F8BT can be assessed from the band edge offsets against electron donors that are known to facilitate dissociation of F8BT excitons, and is generally considered to be at most $0.5 \mathrm{eV}$ [7]. The inverse quadratic dependence of the exciton binding energy on $\varepsilon$ within the Wannier effective-mass exciton model suggests over $60 \%$ reduction in exciton binding energy, or from $0.5 \mathrm{eV}$ to $0.18 \mathrm{eV}$. The exciton delocalization $(\sim \varepsilon)$ will likewise increase, and the singlet-triplet exchange energy will be

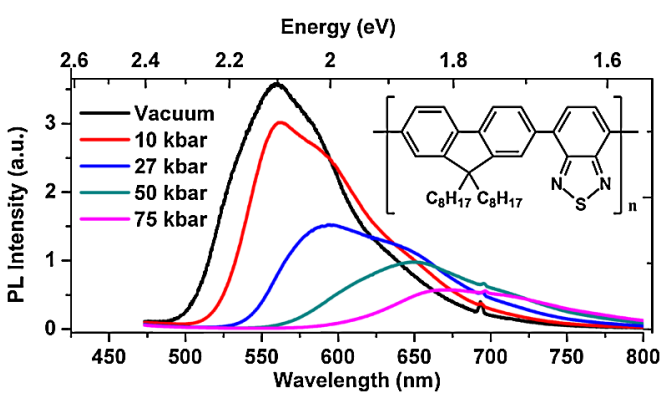

FIG. 1 (color online). Steady-state PL measurements at $407 \mathrm{~nm}$ excitation at various pressures. The PL spectrum in vacuum was measured on a spectrosil substrate and was rescaled to match the increase in PL intensity when releasing the pressure. The inset shows the structure of F8BT. 
reduced. We caution that the Wannier exciton model was intended for inorganic semiconductors, so quantitative agreement is not expected for organic materials. We note that recent reflectivity measurements on films of poly( $p$-phenylene vinylene) show a 30\% increase in the dielectric constant perpendicular to the chain direction with pressure [11].

Absorption and emission spectroscopies reveal a reduction of the optical band gap and reduced fluorescence intensities for almost all semiconducting polymers studied under pressure [12-16]. Loi et al. invoke the photogeneration of nonemissive polarons to account for reduced emission in compressed sexithiophene crystals; however, polarons cannot be directly detected via emission measurements [17]. In contrast, ultrafast transient absorption (TA) spectroscopy can probe the formation of nonemissive states. Pressure-dependent TA has been carried out on films of polythiophene [14]. However, since those measurements employed probes tuned to discrete wavelengths, the dynamics of different photoexcitations was convoluted with pressure-dependent spectral shifts.

Here, we present the first broadband TA spectroscopy investigation of an organic semiconductor under pressure. This is a challenging experiment because, without the sensitivity advantages of using single channel lock-in detection, it is difficult to resolve transient optical signals at sufficiently low excitation density to avoid the artefacts of second order interactions [18]. The challenge is compounded for high-pressure samples because the narrow aperture $(250 \mu \mathrm{m})$ of the diamond anvil cell reduces the effective sample volume and places severe constraints on the optical configuration.

The TA setup was based on that described in [19]. An optical parametric amplifier (OPA, TOPAS) tuned to $490 \mathrm{~nm}$ was used as the excitation source, and the broadband output of a noncollinear OPA spanning from around $500-780 \mathrm{~nm}$ was used as a probe. To achieve time resolution down to the optical pulse length of $\sim 100 \mathrm{fs}$, the probe pulse was delayed via a mechanical delay stage before being overlapped collinearly by a dichroic beam splitter. The use of a diverging pump pulse at the sample position ensured optimal pump-probe overlap and minimized reexcitation via reflection from the backside of the diamond.

A $512 \mathrm{~nm}$ cutoff filter was used to filter the residual pump pulse before the spectrometer. The additional optics and the anvil diamond caused a group velocity dispersion of $\sim 1 \mathrm{ps}$ for the broadband probe, which was corrected during data processing and thus did not reduce the time resolution. We note that strong TA signals from nitrogen impurities in the diamonds prevented the use of excitation wavelengths below $\sim 450 \mathrm{~nm}$. The nitrogen impurities were not problematic for PL measurements, as we did not detect any emission signal.

All samples were prepared via spin coating a film of F8BT onto a substrate coated with a sacrificial water-soluble polymer. The F8BT film was floated off in deionized water and transferred onto the anvil diamond. By this method, we achieved homogeneous films with control over thicknesses, as is necessary for absorption measurements. This method further ensured a homogeneous hydrostatic pressure on the sample, as the film is only loosely attached to the diamond surface. The hydrostatic pressure setup consists of a minicryogenic diamond anvil cell (DAC, Diacell Ltd. UK). After loading a film, the DAC was put under vacuum overnight to remove any residual water, purged several times with argon gas to remove any traces of oxygen, and cryogenically loaded with liquid argon as the pressure medium at liquid nitrogen temperature. A small ruby crystal was inserted to allow pressure calibration based on its well-studied fluorescence redshift under pressure [20]. The pressure calibration and PL were taken using a $500 \mathrm{~mm}$ spectrograph (PI-SpectraPro2500i) combined with a CCD camera (PIActon-100F) and a pulsed $407 \mathrm{~nm}$ laser as the excitation source (PicoQuant LDH400 $40 \mathrm{MHz}$ ).

Singlet excitons in F8BT have strong emission and absorption spectral features within our detection range (525-780 nm) that can be used to assess their chargetransfer character $[5,18]$. With broadband TA spectroscopy, we are able to track the pressure-dependent signatures of different populations and select the correct set of wavelengths with which to compare the dynamics as a function of pressure. Under pressure we find that all spectral features are redshifted, stimulated emission subsides to leave chargelike absorption, and excitations are longerlived. Our observations conform with the formation of more delocalized singlet excitons as films are compressed.

Figure 1 shows the pressure-dependent PL. Schmidtke et al. have recently reported PL measurements of F8BT in film and solid-state solutions under hydrostatic pressure [12]. They ascribe the observed redshift in the isolated chains of the solid-state solution to planarization of the polymer backbone, consistent with Raman spectroscopy. The additional redshift of the PL spectra in film is attributed to interchain interactions. The PL spectra in Fig. 1 are in good agreement with those found in [12]. The use of a wide spectral range CCD camera allowed us to quantitatively track the decrease in PL intensity with increasing pressure from vacuum to $75 \mathrm{kbar}$. Figure 1 shows that the $\sim 400 \mathrm{meV}$ redshift is accompanied by a $\sim$ fivefold reduction in PL intensity over this pressure range.

The PL intensity reduction might partly be caused by measurement artefacts such as the changing absorption cross section at the excitation wavelength $(407 \mathrm{~nm})$. Based on the absorption measurement at ambient pressure and the expected redshift of the absorption spectrum by $\sim 0.6 \mathrm{meV} / \mathrm{kbar}$ [12], we expect that this effect could cause a reduction in the PL intensity by up to $30 \%$. Second, because the excitation energy changes relative to the band gap, we create excitons with higher excess energy at high pressure. We can explicitly exclude the possibility that degradation accounts for decreasing PL intensities, as we saw a full recovery of the PL intensity with decreasing pressure. Other minor effects that we have not accounted for are the out-coupling efficiency and the changing sample 
position caused by a contraction of the pressure cell. Nevertheless, the reduction in PL intensity is much stronger than these artefacts combined. The reduced PL intensity under pressure is consistent with previous literature $[14,17]$.

The TA measurements were carried out under low excitation fluence of $\sim 5 \mu \mathrm{J} / \mathrm{cm}^{2}$, where decay kinetics are independent of fluence [18]. Figure 2 shows the evolution of TA spectra at pressures between vacuum and $75 \mathrm{kbar}$ for different delay times. In vacuum, the spectral range only covers the stimulated emission (SE) at 500-600 nm and the exciton photoinduced absorption (PA) at $625-800 \mathrm{~nm}$. Over the first 100 ps the SE signal shows a dynamic redshift which was previously ascribed as exciton hopping to lower-energy sites [21]. The SE signal clearly overlaps with the steady-state PL spectrum.

At hydrostatic pressure of $27 \mathrm{kbar}$, the early-time spectrum shows two positive signals: (i) a SE signal from around $570 \mathrm{~nm}$ to $620 \mathrm{~nm}$ that overlaps with the PL spectrum, and (ii) the steep edge of the ground-state bleach (GSB) signal below $550 \mathrm{~nm}$ since the GS absorption has shifted into our detection window at this pressure. The GSB shows similar decay dynamics to the PA at $750 \mathrm{~nm}$, whereas the SE signal disappears within $\sim 100 \mathrm{ps}$. After this time the SE is replaced by the exciton PA signal. At $75 \mathrm{kbar}$, the entire positive signal can be accounted for by the GSB and has no overlap with the PL spectrum. The absence of a dynamically shifting SE contribution accounts for the isosbestic point observed at $620 \mathrm{~nm}$. The region of the corresponding PL spectrum where we would expect a positive SE band shows an unusually blueshifted, broad, featureless PA band. These spectral properties are very atypical for singlet excitons in polymer films. In contrast, these features are commonly observed for nonemissive or

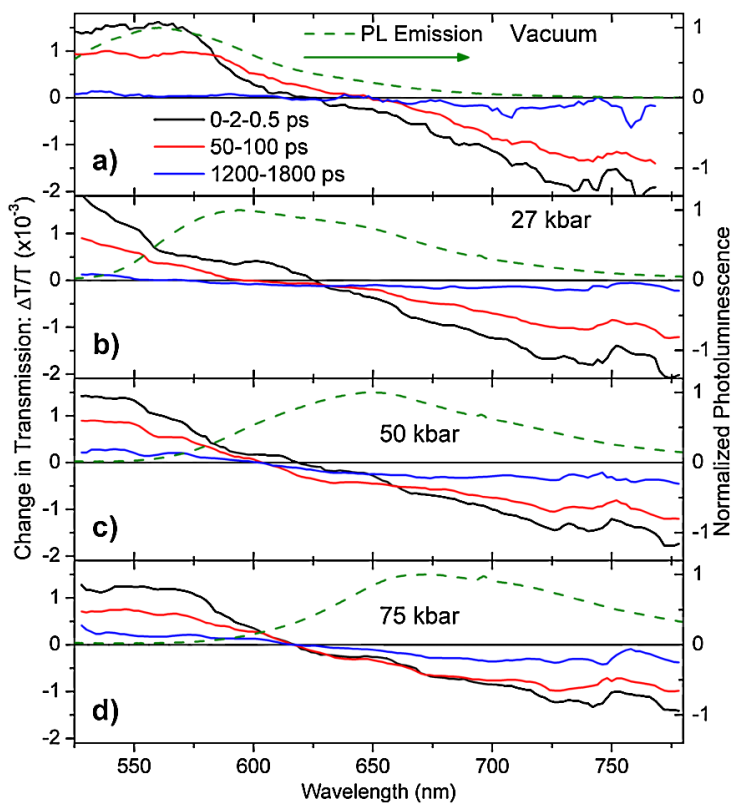

FIG. 2 (color online). TA spectra at various pressures and delay times as indicated. The dashed lines show the normalized PL spectra for comparison. only weakly emissive charge-transfer (CT) excitons [19]. For CT excitons or charge pairs, we expect a broad PA band right below the band gap.

Figure 3(a) shows TA kinetics for the spectra at $50 \mathrm{kbar}$ [Fig. 2(c)] for the GSB, the PA, and in a region where SE and exciton PA compete. The delocalization of the singlet excitons in the first 100 ps reduces the positive SE signal; thus, we see an increase in the negative PA signal at 620$650 \mathrm{~nm}$. After about $200 \mathrm{ps}$ the TA spectra do not change further, and all three kinetic traces decay at the same rate, confirming the presence of only one excitonic species.

To study the effect of pressure on the decay times of the excited states, we investigated the decay kinetics of the exciton PA signal in the range $700-800 \mathrm{~nm}$, as shown in Fig. 3(b). We emphasize that it was necessary to redshift the integrated wavelength region to track the pressureinduced spectral shifts and avoid contribution from the SE, highlighting the value of our broadband detection scheme. Under increased pressure, the decay of the PA signal slows down considerably beyond 100 ps. The reduced PL yield shown in Fig. 1 therefore results not from more rapid nonradiative decay channels, but instead from the reduced radiative decay rate of the delocalized highpressure exciton. A strongly reduced radiative decay rate was also found for poly(di-octylfluorene) (F8), as shown in the SI [22]. This suggests that pressure delocalization is not specific to donor-acceptor copolymers. We therefore expect that the higher screening causes a delocalization over more repeat units along the chain and over adjacent chains of the high-pressure exciton. The interchain delocalization will be further increased by the reduced interchain distance. We believe that the pressure-induced increased orbital overlap in F8BT by backbone planarization has a generally weaker localizing effect than the dielectric delocalization. The interplay of these two effects is the most

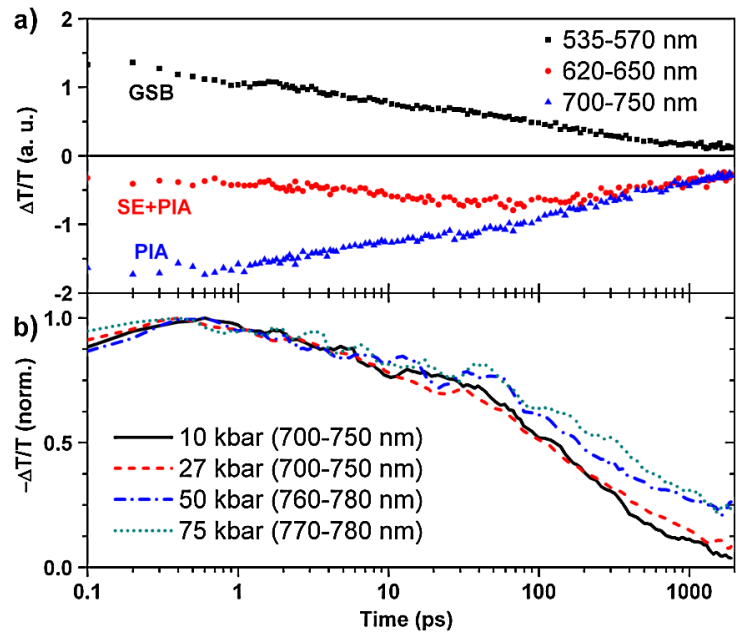

FIG. 3 (color online). (a) TA kinetics for three distinct wavelength regions at $50 \mathrm{kbar}$ (rescaled to emphasize their differences). (b) Decay dynamics of the normalized PA signal for various pressures. The slight rise at $\sim 20 \mathrm{ps}$ is due to reexcitation via reflection from the backside of the diamond. 

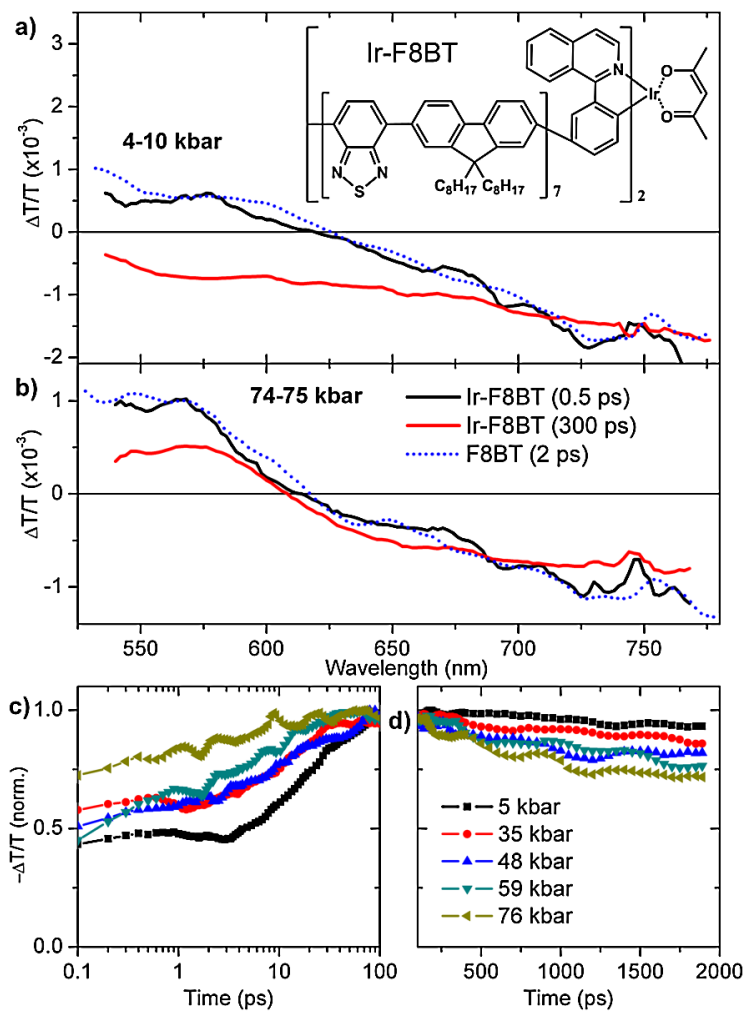

FIG. 4 (color online). Structure of Ir-F8BT (inset) and TA spectra of the photoexcited states in Ir-F8BT and F8BT for (a) $\sim 10 \mathrm{kbar}$ and (b) $\sim 75 \mathrm{kbar}$. TA kinetics of the PA band at different pressures (c) for the first $100 \mathrm{ps}$ on a log scale and (d) for 100-2000 ps on a linear scale.

likely explanation for the emergence of both a faster and a slower PL decay component found for F8BT under increased pressure [12]. Without the complications of donor-acceptor character, the PL decay in F8 simply becomes longer under pressure.

We further used this setup to measure the effect of pressure on triplet excitons. An Ir-F8BT oligomer can be used to populate the F8BT triplet level [19]. At low pressure the initial photoexcitations at 0.5 ps in Fig. 4(a) are singlet excitons. The strong spin-orbit coupling results in fast intersystem crossing within $10 \mathrm{~s}$ of ps. The resultant triplet excitons (at $300 \mathrm{ps)} \mathrm{show} \mathrm{a} \mathrm{broader} \mathrm{PA} \mathrm{band} \mathrm{than} \mathrm{the}$ initial singlet excitons in F8BT or Ir-F8BT. At high pressure [Fig. 4(b)] the spectral evolution of the photoexcited states is more subtle and the TA spectra closely resemble that of high-pressure excitons in F8BT. Figures 4(c) and 4 (d) show the TA kinetics of the integrated PA for different pressures. The increase of PA intensity in the first $100 \mathrm{ps}$ represents the intersystem crossing and associated loss of SE, whereas the following 2 ns show a slow decay of the triplet excitons. At all pressures the resultant photoexcitation persists much longer than the photoexcited states in F8BT, which confirms that the triplet formation mechanism is still functioning at high pressure. We find though that the triplet excitons decay significantly faster at high pressure. One contributing mechanism may be that the pressure-induced reduction in singlet-triplet exchange energy allows thermal excitation back to the singlet manifold. We note that the decrease in triplet lifetime is in contrast to the increase of the singlet exciton with pressure. Both are caused by the increase in dielectric screening and associated delocalization and exchange energy reduction.

In summary, the TA spectra of F8BT under hydrostatic pressure show that SE is replaced by excited state absorption below the band gap. These effects are generally observed for chargelike excited states. In our case the photoexcitations at high pressure take the form of more delocalized excited states, which slows down radiative decay processes as observed by the slower decay kinetics and the reduced fluorescence efficiency at high pressure. These findings can be accounted for by the increase of the dielectric constant with pressure.

The authors thank Ian Howard and Roger Beadle for useful discussions and technical assistance. S. A.-S. thanks the Ernest Oppenheimer Foundation for financial support.

*sa468@cam.ac.uk

†resent address: MacDiarmid Institute for Advanced Materials and Nanotechnology, New Zealand.

*Present address: Max Planck Institut für Polzmerforschung, Mainz, Germany.

§rhf10@cam.ac.uk

[1] A. Köhler and D. Beljonne, Adv. Funct. Mater. 14, 11 (2004).

[2] J. J. M. Halls et al., Nature (London) 376, 498 (1995).

[3] C. W. Tang, Appl. Phys. Lett. 48, 183 (1986).

[4] G. Yu et al., Science 270, 1789 (1995).

[5] C.L. Donley et al., J. Am. Chem. Soc. 127, 12890 (2005).

[6] J. J. M. Halls et al., Adv. Mater. 12, 498 (2000).

[7] A. C. Morteani et al., Adv. Mater. 15, 1708 (2003).

[8] G. Heimel et al., J. Phys. Condens. Matter 15, 3375 (2003).

[9] J. Mårdalen et al., J. Phys. Condens. Matter 10, 7145 (1998).

[10] C. M. Ramsdale and N. C. Greenham, Adv. Mater. 14, 212 (2002).

[11] V. Morandi et al., J. Appl. Phys. 107, 073106 (2010).

[12] J. P. Schmidtke et al., Phys. Rev. Lett. 99, 167401 (2007).

[13] V. Morandi et al., Phys. Rev. B 79, 045202 (2009).

[14] B. C. Hess, G. S. Kanner, and Z. V. Vardeny, Phys. Rev. B 47, 1407 (1993).

[15] C. M. Martin et al., Phys. Rev. B 68, 115203 (2003).

[16] M. Chandrasekhar et al., Synth. Met. 119, 657 (2001).

[17] M. A. Loi et al., Phys. Rev. Lett. 86, 732 (2001).

[18] M. A. Stevens et al., Phys. Rev. B 63, 165213 (2001).

[19] S. Westenhoff et al., J. Am. Chem. Soc. 130, 13653 (2008).

[20] G. J. Piermarini et al., J. Appl. Phys. 46, 2774 (1975).

[21] L. M. Herz et al., Phys. Rev. B 70, 165207 (2004).

[22] See supplementary material at http://link.aps.org/ supplemental/10.1103/PhysRevLett.105.195501 for timeresolved fluorescence measurements on F8 under pressure. 